\title{
BOAS PRÁTICAS DE ENFERMAGEM NA UNIDADE DE TERAPIA INTENSIVA: DESENVOLVENDOO HISTÓRICO DE ENFERMAGEM
}

Marisa Gomes dos Santos ${ }^{1}$

Tatiana Gaffuri da Silva²

Alexsandra Martins da Silva²

Júlia Valéria de Oliveira Vargas Bitencourt ${ }^{1}$

Eliane Regina Pereira do Nascimento ${ }^{2}$

Katia Cilene Godinho Bertoncello²
ORCID: https://orcid.org/0000-0003-4776-9928

ORCID: https://orcid.org/0000-0001-6854-0319

ORCID: https://orcid.org/0000-0001-9147-9990

ORCID: https://orcid.org/0000-0002-3806-2288

ORCID: https://orcid.org/0000-0002-0484-7458

ORCID: https://orcid.org/0000-0002-2518-3136

Objetivo: Analisar o significado da prática do histórico de enfermagem em uma Unidade de Terapia Intensiva. Método: Estudo qualitativo, realizado em uma Unidade de Terapia Intensiva do sul do Brasil, com nove enfermeiros. A coleta de dados ocorreu por meio de grupo de discussão, tendo como questão disparadora: O que significa a prática do Histórico de Enfermagem na Unidade de Terapia Intensiva? Os dados foram analisados por meio do Discurso do Sujeito Coletivo. Resultados: O estudo deu origem a três discursos com as ideias centrais: $O$ histórico de enfermagem representa a qualificação do cuidado e o empoderamento da enfermagem; $A$ informação como ferramenta do cuidado; $O$ instrumento utilizado para o histórico de enfermagem como construção coletiva. Considerações Finais: $O$ histórico de enfermagem como boa prática confere à profissão, autonomia, empoderamento e visibilidade. Ademais, qualifica e assegura o cuidado oferecido respaldando o exercício profissional.

Descritores: Processo de enfermagem; Unidades de Terapia Intensiva; Cuidados de Enfermagem; Autonomia profissional.

\section{THE HISTORY OF NURSING AS GOOD PRACTICE IN THE INTENSIVE THERAPY UNIT}

Objective: To analyze the meaning of nursing practice in an Intensive Care Unit. Method: Qualitative study, conducted in an Intensive Care Unit in the south of Brazil, with nine nurses. The data collection took place through a discussion group, having as a triggering question: What does the practice of Nursing History in the Intensive Care Unit? The data were analyzed through the Collective Subject Discourse. Results: The study gave rise to three discourses with the central ideas: Nursing history, nursing qualification and empowerment; Information as a care tool; The nursing history as a collective construction. Final Considerations: The nursing history as a good practice confers on the profession, autonomy, empowerment and visibility.

Descriptors: Nursing Process; Intensive Care Units; Nursing Care; Professional Autonomy.

\section{EL HISTÓRICO DE ENFERMERÍA COMO BUENA PRÁCTICA EN LA UNIDAD DE TERAPIA INTENSIVA}

Objectivo: Analizar el significado de la práctica del historial de enfermería en una Unidad de Terapia Intensiva. Método: Estudio cualitativo, realizado en una Unidad de Terapia Intensiva del sur de Brasil, con nueve enfermeros. La recolección de datos ocurrió por medio de un grupo de discusión, teniendo como cuestión desencadenadora: Qué significa la práctica del Historial de Enfermería en la Unidad de Terapia Intensiva? Los datos fueron analizados por medio del Discurso del Sujeto Colectivo. Resultados: El estudio dio origen a tres discursos con las ideas centrales: El histórico de enfermería, la calificación del cuidado y el empoderamiento de la enfermería; La información como herramienta del cuidado; El histórico de enfermería como construcción colectiva. Consideraciones finales: El historial de enfermería como buena práctica confiere a la profesión, autonomía, empoderamiento y visibilidad.

Descriptores: Proceso de Enfermería; Unidades de Cuidados Intensivos; Atención de Enfermería; Autonomía Profesional.

Universidade Federal da Fronteira Sul

2Universidade Federal de Santa Catarina

Autor correspondente: Alexsandra Martins da Silva E-mail: alexsandrams.enf@gmail.com

Recebido: 19/05/2019

Aceito: 08/07/2019 


\section{INTRODUÇÃO}

O Processo de Enfermagem (PE), como instrumento metodológico, guia as ações profissionais da enfermagem e possibilita um cuidado coerente com as necessidades individuais e coletivas de saúde do ser humano. Permite, aos profissionais enfermeiros, avaliação clínica necessária para um cuidar permeado pelo conhecimento científico, ético e humanizado. Organiza e assegura a continuidade das informações acerca do cuidado prestado pela equipe de enfermagem, favorecendo a evolução da assistência, de acordo com os resultados esperados para a recuperação do paciente ${ }^{(1-2)}$.

Como tecnologia do cuidado, o PE está organizado em cinco etapas inter-relacionadas: Histórico de Enfermagem (HE); Diagnóstico de Enfermagem (DE); Planejamento de Enfermagem; Implementação e Avaliação de Enfermagem ${ }^{(3-4)}$. O HE, como primeira etapa do PE, oferece as informações para o julgamento, planejamento e delimitação das intervenções de enfermagem, subsidiando as etapas subsequentes, viabilizando a tomada de decisão e qualificando a assistência oferecida nos diversos contextos do sistema de saúde(5).

O HE e demais etapas do PE, como boa prática em enfermagem, aprimoram os resultados encontrados, a qualidade e consistência dos cuidados oferecidos, remodelando $\mathrm{o}$ atendimento e a prática profissional. A ênfase do PE, apesar de existir há décadas, tem relação com sua subutilização no cotidiano dos serviços como ferramenta e método no desempenho das ações de enfermagem e, em especial, com o interesse crescente dos profissionais enfermeiros em adotarem ações coerentes com as necessidades individuais e coletivas de saúde dos seres humanos, através da criação, implantação e gestão de políticas públicas, organização e humanização do cuidado, redução e controle de riscos e qualificação da atenção prestada a fim de obter melhores resultados e reconhecimento profissional ${ }^{(6-7)}$.

As boas práticas em enfermagem constituem-se pela busca por ações que garantam que os serviços prestados estejam dentro dos padrões de qualidade exigidos para os fins a que se propõem ${ }^{(8)}$. Neste sentido, o PE reforça o escopo da enfermagem, com resultados positivos no que se refere à redução de danos, menor permanência no ambiente hospitalar, cuidado seguro, prática clínica segura alicerçada no conhecimento científico e resultados que fortalecem a profissão e sua proposta de cuidado integrado, expandido e complexo.

Ao utilizá-lo na Unidade de Terapia Intensiva (UTI), ambiente destinado ao cuidado de pessoas em estado crítico de saúde com instabilidade clínica e risco de morte, o PE vai ao encontro do fortalecimento da ciência em enfermagem, com resultados imediatos favoráveis ao paciente e ao longo do tempo ao sistema de saúde ${ }^{(9)}$.

\section{OBJETIVO}

Analisar o significado da prática do histórico de enfermagem em uma Unidade de Terapia Intensiva.

\section{MÉTODO}

\section{Tipo de Estudo}

Estudo descritivo, exploratório e com abordagem qualitativa. Estudos qualitativos têm como propósito compreender o fenômeno através de uma abordagem flexivel e permissão da livre expressão das informações, experiências e vivências dos participantes ${ }^{(12)}$.

\section{Local do estudo}

Realizado em uma UTI, situada no sul do Brasil, com 17 leitos e nove enfermeiros, sendo oito assistenciais e um coordenador geral. O HE foi implantado na UTI no ano de 2016, sustentado pelo referencial teórico das Necessidades Humanas Básicas (NHB) de Wanda de Aguiar Horta ${ }^{(11)}$.

\section{Referencial teórico-metodológico}

O estudo utilizou a Teoria das Necessidades Humanas Básicas de Wanda de Aguiar Horta, baseada no modelo da motivação humana de Maslow e na classificação das necessidades, adotada por João Mohana (necessidades psicobiológicas, psicossociais e psicoespirituais), tendo como princípios o respeito à unicidade do sujeito e o cuidado centrado no ser humano. Contudo, para este estudo, enfocou-se a sequência das necessidades, identificando os problemas e respeitando as conclusões diagnósticas ${ }^{(11)}$.

\section{Coleta dos dados}

Participaram do estudo enfermeiros da UTI que atenderam aos critérios de elegibilidade com atuação na UTI por mais de seis meses, e prática no HE por no mínimo noventa dias. Como critério de exclusão, considerou-se estar em licença ou afastamento no momento da coleta de dados. Dos nove enfermeiros, apenas um não atendeu aos critérios estabelecidos, sendo assim participaram do estudo oito profissionais.

A coleta de dados se deu no período entre março a maio de 2018, por meio de encontro do grupo de discussão, que ocorreu em sala de estudos do próprio hospital, com duração 
de 120 minutos. Todos os nove enfermeiros do estudo assinaram o Termo de Consentimento Livre e Esclarecido. A questão disparadora foi: $\mathrm{O}$ que significa a prática do $\mathrm{HE}$ na UTI para vocês? O encontro foi gravado, transcrito na integra e posteriormente validado pelos enfermeiros.

\section{Procedimentos de análise dos dados}

Os dados foram analisados por meio do Discurso do Sujeito Coletivo (DSC). Trata-se de uma técnica de tabulação e organização de dados qualitativos, desenvolvida por Lefevre e Lefevre, utilizada em pesquisas da área da saúde. Possibilita conhecer os pensamentos, representações, crenças e valores de uma coletividade sobre um determinado tema, tendo como proposta a reconstituição do ser coletivo e opinante na forma de um sujeito de discurso emitido na primeira pessoa do singular ${ }^{(13)}$.

A técnica contempla quatro figuras metodológicas, as expressões chaves (ECHs), trechos do discurso original que representam o conteúdo da questão em análise, as ideias centrais (ICs) que compõem os sentidos das expressões chaves, a Ancoragem (AC), considerada como expressão teórica, ideologia ou crença e por último, o Discurso do Sujeito Coletivo (DSC), definido como discurso síntese ${ }^{(13)}$.

Aorganização dos Discursos se deu por meio de repetidas leituras e grifo das partes relevantes dos depoimentos, consideradas as ECH. Na sequência, foram identificadas as ideias centrais e organizado os discursos sintese. A ancoragem não foi utilizada.

A análise dos dados deu origem a três discursos com as ideias centrais (IC) ICl: O Histórico de enfermagem representa a qualificação do cuidado e o empoderamento da enfermagem, IC2: A informação como ferramenta do cuidado, IC3: O instrumento utilizado para o histórico de enfermagem como construção coletiva. Após a descrição de cada discurso no item resultados, os participantes foram identificados com letra $E$, referente a entrevistado e a sequência numérica correspondente a ordem de transcrição dos registros do grupo focal.

\section{Procedimentos éticos}

Estudo aprovado pelo Comitê de Ética em Pesquisa da Universidade Federal da Fronteira Sul (CEP-UFFS), com parecer número 1.677.677. Após aprovação, o pesquisador apresentou aos participantes o desenho metodológico e objetivo, obtendo o voluntário consentimento, assegurando a confidencialidade e proteção dos dados, conforme propõe o disposto na Resolução do Conselho Nacional de Saúde (CNS) no 466/2012(10)

\section{RESULTADOS}

Dos 8 (100\%) enfermeiros que participaram do estudo, 7 (87.5\%) são do sexo feminino e 1 (12.5\%) do sexo masculino, com predomínio da faixa etária de 26 a 41 anos. A maioria dos profissionais tem especialização em terapia intensiva e o tempo de atuação nesta área variou de dois anos e seis meses a 18 anos.

O DSCl foi organizado a partir dos depoimentos de sete participantes, e destaca o HE como representação de qualidade no cuidado e empoderamento profissional.

ICl: O Histórico de Enfermagem representa a qualificação do cuidado e o empoderamento da enfermagem.

DSC 1- O paciente internado na UTI e a gente não sabia nada dos antecedentes dele, desde o histórico familiar, onde e com quem morava, se já havia feito cirurgia, se tomava medicação em casa, se não tomava. Agora, durante a entrevista, uma pergunta puxa outra, e vamos descobrindo dados e informações importantes sobre o paciente. Sabemos se o paciente é ou não tabagista, se usa álcool, as medicações que faz uso, se têm outras doenças, nossa, melhorou muito! Agora podemos dizer que com a implantação do roteiro do $H E$, conhecemos melhor o paciente, e com isso, melhorou o atendimento e o cuidado oferecido, ficamos fortalecidos. (E2, E3, E4, E5, E6, E7, E8)

O DSC2 se constituiu dos depoimentos de cinco participantes e evidencia a importância do registro no cuidado dispensado ao paciente e família.

IC2: A informação como ferramenta do cuidado.

DSC 2- Agora tem o registro, é uma ferramenta que todos os colegas têm acesso, antes só a gente sabia o que o paciente ou família falavam no momento da internação, porque muitas vezes esquecemos de passar no plantão, e a história do paciente era perdida, o colega ficava sem saber informações importantes, e muitas vezes precisava perguntar novamente para a família. Agora, o histórico serve de referência também para os outros profissionais de saúde, quando alguém precisa de informações, vai lá no histórico e tem acesso. Esse instrumento ficou muito bom, é nossa referência profissional. (E1, E2, E3, E7, E8)

O DSC3 se constituiu dos depoimentos de seis participantes e retrata a importância da participação coletiva na construção do histórico, com o envolvimento 
de profissionais de enfermagem da instituição hospitalar e das instituições de ensino, com sentimento de satisfação durante a construção e implementação.

Ideia central (IC): O instrumento utilizado para o histórico de enfermagem como construção coletiva.

DSC 3- O HE foi pensado pelo coletivo, em um grupo grande, com professores, enfermeiros, estudantes, técnicos, então, não foi só uma pessoa, alguém que chegou com o HE, que pegou algo pronto, a gente fez ele por completo, o grupo construiu junto, foi debatido, discutido, pensado na nossa realidade, no que a gente vivenciava. E foi embasado nos domínios da NANDA e pensado na nossa prática, meu Deus, foi torcido o liuro na nossa cabeça, e acho que isso tem feito toda a diferença. Foi muito trabalhoso, mas valeu! Fico muito feliz em fazer parte desta construção, está sendo uma referência para todo hospital, é nosso referencial de unidade, hoje ele já faz parte, é nosso, faz parte da nossa história! (E1, E2, E5, E6, E7, E8)

\section{DISCUSSÃO}

O discurso com a Ideia Central (IC) "O Histórico de Enfermagem (HE), a qualificação do cuidado e o empoderamento da enfermagem", evidencia a importância do HE no processo de cuidado, como prática científica que empodera o profissional frente à tomada de decisão e a relação estabelecida com a equipe de saúde e familia, alinhado as concepções das boas práticas em saúde.

Ao implantar o HE, e posteriormente as demais etapas do PE, há avanços na autonomia e nas relações de poder da enfermagem. Sua prática possibilita ao enfermeiro aplicar seus conhecimentos, sua expertise e sua responsabilidade profissional pela conquista de um cuidado qualificado que inclui e valoriza o modo de pensar e as decisões tomadas pelos pacientes nas situações que the dizem respeito. Associando assim, o trabalho desenvolvido a cientificidade e a expansão em saúde ${ }^{(14)}$.

Nesta perspectiva do registro do $\mathrm{HE}$, o enfermeiro acessa a informação com mais facilidade, agiliza a tomada de decisão e melhora o desempenho de suas funções ${ }^{(15)}$. Implica também em parceria entre o profissional enfermeiro, a equipe de enfermagem, paciente e família, com definição conjunta de prioridades no cuidado e ampliação do foco de atuação, através do cuidado holístico, que vai além das exigências fisiopatológicas ${ }^{(16)}$.

Assim sendo, o exercício do HE vai ao encontro da restauração da saúde individual e coletiva e em especial do fortalecimento da enfermagem como ciência do cuidado com a qualificação da assistência e o empoderamento da enfermagem. O HE também é percebido como uma ferramenta de boa prática no serviço em foco. Para tanto, destaca-se nas concepções das boas práticas, o uso de metodologias, que por meio da experiência e ou investigação, remetem a uma confiabilidade comprovada na obtenção de resultados assertivos ${ }^{(17)}$.

O segundo discurso, com a IC "A informação como ferramenta do cuidado", revela a importância do registro diário para a continuidade do trabalho desenvolvido pela equipe de enfermagem, seus pares e demais integrantes da equipe multiprofissional oportunizando visibilidade do trabalho do enfermeiro, bem como, a articulação interdisciplinar.

No contexto hospitalar, os dados contidos no prontuário do paciente representam importante fonte de comunicação entre os profissionais envolvidos no cuidado, expõe as necessidades e situações de saúde dos pacientes, tal qual, as condutas clínicas que foram implementadas e a avaliação contínua do paciente e do cuidado, sendo percebido como responsabilidade profissional e social(18).

Implica no processo de satisfação do paciente, família e equipe ${ }^{(19)}$, imprescindivel para legitimar as ações profissionais junto ao usuário, a família e servir de fonte de informações para questões jurídicas, de pesquisas e de educação(20-21).

Ainda, no que tange a visibilidade expressa na IC 2, relacionada ao uso do $\mathrm{HE}$, fortalece as evidências por explicitar a relação estabelecida entre o conhecimento, a prática e as escolhas feitas pelos pacientes e família(22). Estudo desenvolvido sobre o trabalho da enfermagem e sua visibilidade, aponta que os enfermeiros reconhecem que o conhecimento clínico sustentado pelas etapas do PE, com base em seus Sistemas de Linguagem Padronizados (SLP), valoriza o trabalho desenvolvido, por expressar cientificidade, além de permitir aos outros profissionais, pacientes e usuários, entre outros, a compreensão que as intervenções de enfermagem são resolutivas e expressam cientificidade ${ }^{(23)}$. Com isso, todo este empenho, organização, aplicação de estratégias e ferramentas de cuidado como o $\mathrm{HE}$, corroboram com as melhores práticas em saúde.

$\mathrm{O}$ discurso com a IC "O instrumento utilizado para o histórico de enfermagem como construção coletiva", destaca a inovação de implementar o HE no cuidado ao paciente crítico e o envolvimento do grupo para alinhamento de ideias e apropriação deste recurso no cotidiano de trabalho. A construção compartilhada entre os profissionais do serviço, os gestores e academia foi evidenciada, como potencialidade para a troca de experiências, sensação de pertencimento, motivação e valorização dos diferentes saberes ${ }^{(24)}$. 
O trabalho em grupo fortalece as relações estabelecidas entre os partícipes, aproximando e aprimorando a capacidade de respeitar as divergências e diferenças individuais, além de possibilitar o reconhecimento de lacunas, que podem ser de conhecimento ou de desenvolvimento de práticas, e mobilizar a busca de novas estratégias para a transformação da realidade(25).

Ademais, em espaços formados por uma coletividade de pares, veiculam discussões sobre eventos que pertencem aos cotidianos de trabalho, que ao emergirem como ponto de debate são passiveis de redefinirem posturas e a forma de agir dos sujeitos enquanto profissionais, transformando práticas dominantes por outras mais resolutivas e eficazes no dia a dia do cuidado.

A proposta participativa, revela uma dinâmica de trabalho divergente das imposições verticais hegemônicas da maioria das instituições, propondo espaços de fortalecimento do coletivo com valorização do indivíduo no grupo(24). No entanto, os desafios e a complexidade desta trajetória, foram evidenciados, como a exigência de conhecimento para além dos saberes pré-existentes, e que não se concluem com a construção e implantação do instrumento, mas sim, exige de todos, um contínuum, no que se refere a conhecimento e empenho.

Conquanto, outras condições foram impostas, como desapegar de velhas práticas da assistência de enfermagem, quebrar paradigmas e alterar o habitual processo de trabalho, difícil até mesmo para aqueles dispostos a mudança(26). $\mathrm{Na}$ mesma perspectiva, é fato que a constante evolução tecnológica do cuidado, em especial no ambiente de UTI, imprime nos profissionais destas unidades a necessidade de acompanhar tais mudanças, o que torna a implantação do $P E$ importante ferramenta frente às vivências diárias da unidade ${ }^{(27)}$

Nessa direção o PE se apresenta como boa prática, assegurando qualidade à assistência oferecida ao paciente e sua família, balizando a conquista da autonomia e buscando romper o paradigma do que é preconizado e o que é realizado no cotidiano da enfermagem, sobremaneira colaborando para o planejamento e organização da prática gerencial e assistencial(28). Neste sentido, autores reforçam que a prática avançada na enfermagem tem se fortalecido em atividades educativas, no desenvolvimento da consulta de enfermagem, na coordenação do trabalho em equipe, desenvolvimento de rotinas administrativas, entre outras ${ }^{(29)}$.

\section{CONSIDERAÇÕES FINAIS}

Os discursos dos profissionais revelaram o $\mathrm{HE}$, como uma tecnologia do cuidado que orienta o raciocínio, melhora a qualidade do cuidado por meio da sistematização da avaliação clínica, com ênfase no fortalecimento da autonomia e empoderamento profissional. Sua utilização, bem como das demais etapas do PE, representam o avanço da enfermagem frente às demandas impostas no dia a dia dos serviços de saúde, bem como, uma das características de boas práticas em enfermagem.

O estudo revela a transformação no modo da Enfermagem cuidar na UTI, na perspectiva de um fazer embasado na ação/reflexão/ação, que visa atender as necessidades gerais e específicas do paciente em cuidados intensivos.

\section{REFERÊNCIAS}

1. Dal Sasso GTM, Barra DCC, Paese F, Almeida SRW de, Rios GC, Marinho MM, Debétio M. Computerized nursing process: methodology to establish associations between clinical assessment, diagnosis, interventions, and outcomes. Rev. esc. enferm. USP [Internet]. 2013 [cited 2018 may 25]; 47(1):242-9. Available from: http://dx.doi.org/10.1590/S0080-62342013000100031

2. Conselho Federal de Enfermagem. Resolução cofen-358, 15 de outubro de 2009. [Internet]. [cited 2018 may 25]. Available from: http://www.cofen.gov.br/resoluo-cofen-3582009_4384.html

3. Alvim ALS. O Processo de Enfermagem e suas Cinco Etapas. Enferm Foco [Internet]. 2013 [cited 2018 may 25]; 4(2):140-1. Available from: http://revista.portalcofen.gov.br/index.php/enfermagem/ article/download/531/214

4. Malucelli A, Otemaler KR, Bonnet M, Cubas MR, Garcia TR. Infor- mation system for supporting the nursing care systematization. Rev Bras Enferm [Internet]. 2010 [cited 2018 may 25]; 63(4):62936. Available from: http://www.scielo.br/pdf/reben/v63n4/20.pdf 5. Ferreira AM, Rocha EN, Lopes CT, Bachion MM, Lopes JL, Barros ALBL. Nursing diagnoses in intensive care: cross-mapping and NANDA-I taxonomy. Rev Bras Enferm [Internet]. 2016 [cited 20185 25]; 69(2): 285-93. Available from: http://www.scielo.br/scielo.php?script=sci_abstractEpid=S0034-71672016000200307Elng=enEnrm=iso

6. Amorim LKA, Souza NVDO, Pires AS, Ferreira ES, Souza MB. Vonk ACRP. O trabalho do enfermeiro: reconhecimento e valorização profissional na visão do usuário. Rev enferm UFPE on line [Internet]. 2017 [cited 2018 May 19]; 11(5):1918-25. Available from: https://periodicos.ufpe.br/revistas/revistaenfermagem/article/ viewFile/23341/18946 
7. Miranda Neto MV, Rewa T, Leonello VM, Oliveira MAC. Advanced practice nursing: a possibility for Primary Health Care? Rev Bras Enferm [Internet]. 2018 [cited 2018 May 19]; 71 (Supl 1): 716-21. [Issue Edition: Contributions and challenges of practices in collective health nursing]. Available from: http://www.scielo.br/pdf/ reben/v7lsl/pt_0034-7167-reben-71-sl-0716.pdf

8. Brasil. Agência Nacional de Vigilância Sanitária. Resolução da Diretoria Colegiada - RDC №. 63 DE 25 DE NOVEMBRO DE 2011. Dispõe sobre os Requisitos de Boas Práticas de Funcionamento para os Serviços de Saúde. Available from: file:///C:/Users/Windows\%207/Downloads/RDC_2011-63\%20(1).pdf

9. Backes MTS, Erdmann AL, Büscher A. O ambiente vivo, dinâmico e complexo de cuidados em Unidade de Terapia Intensiva. Rev. Latino-Am. [Internet]. 2015 [cited 2018 May 31]; 23(3):411-8 Available from: http://www.scielo.br/pdf/rlae/v23n3/pt_0104-1169-rlae-0568-2570.pdf

10. Brasil. Conselho Nacional de Saúde. Resolução no 466, de 12 de dezembro de 2012. Available from: http://bvsms.saude.gov.br/bvs/ saudelegis/cns/2013/res0466_12_12_2012.htm

11. Horta WA. Processo de Enfermagem. Editora Guanabara Koogan. Rio de Janeiro, 2019.

12. Polit DF, Beack CT. Prática de enfermagem baseada em evidências. In: Fundamentos da pesquisa em enfermagem: avaliação de evidências para a prática de enfermagem. 7ạ Ed. Porto Alegre: Artmed, 2011.

13. Lefevre F, Lefevre AMC. O sujeito coletivo que fala. Interface (Botucatu). [Internet]. 2006 [citado 2017 Mar 15]; 10(20):517-4. Available from: http://www.scielo.br/pdf/icse/vlOn20/17.pdf

14. Soares MI, Resck ZMR, Terra FS, Camelo SHH. Sistematização da assistência de enfermagem: facilidades e desafios do enfermeiro na gerência da assistência. Esc. Anna Nery [Internet]. 2015 [cited 2018 may 18]; 19(1)47-53. Available from: http://www.scielo. br/pdf/ean/v19nl/1414-8145-ean-19-01-0047.pdf

15. Smith LM, Andrusyszyn MA, Laschinger HKS. Effects of workplace incivility and empowerment on newly graduated nurses organizational commitment. Journal of Nursing Management. [Internet]. 2010 [cited 2018 may 18]; 18(8): 1004-15. Available from: https://sci-hub.tw/10.1111/j.1365-2834.2010.01165.x

16. Kamille RS, Igho LNC, Antônio GAP. Implementação da sistematização da assistência de enfermagem em clínica médico-cirúrgica: limites e possibilidades. Suplemento - Artigos de revisão [Internet]. 2016 [cited 2018 may 18]; 37-44. Available from: https:// periodicos.ufsm.br/revistasaude/article/view/15053

17.Organização Mundial da Saúde - OMS. Guia para a documentação e Partilha das Melhores Práticas em Programas de Saúde. OMS - Escritório Regional Africano Brazzaville; [Internet]. 2008 [cited 2018 may 18]; Available from: http://afrolib.afro.who.int/documents/2009/pt/GuiaMelhoresPratica.pdf

18. Diniz SOS, Silva PS, Figueiredo NMA, Tonini T. Qualidade dos registros de enfermagem: reflexões analíticas em suas formas e conteúdos. Rev Enferm UFPE [Internet]. 2015 [cited 2018 may 18]; 9(10):9616-23. Available from: file:///C:/Users/Windows\%207/ Downloads/10908-23668-1-PB.pdf

19. Barral LNM, Ramos LH, Vieira MA, Dias OV, Sousa LPS. Análise dos registros de enfermagem em prontuários de pacientes em um
Hospital de Ensino. Rev. Min. Enferm. [Internet]. 2012 [cited 2018 may 25]; 16(2):188-93. Available from: http://www.scielo.br/pdf/ ean/v19nl/1414-8145-ean-19-01-0047.pdf

20. Barbosa SF, Tronchin DMR. Manual for monitoring the quality of nursing home care records. Rev Bras Enferm. [Internet]. 2015 [cited 2018 may 25]; 68(2):253-60. Available from: http://dx.doi.or$\mathrm{g} / 10.1590 / 0034-7167.2015680210$

21. Assunção RC. Dalri MCB. Avaliação dos aspectos éticos e legais dos registros de enfermagem. Ciênc Cuid Saúde [Internet]. 2010 [cited 2018 May 26]; 9(4):676-81. Available from: http:// www.periodicos.uem.br/ojs/index.php/CiencCuidSaude/article/ view/13812/7184

22. Frota LA, Camponogara S, Arboit EL, Tolfo F, Beck CLC, Freitas EO. Rev. Eletr. Enf. [Internet]. 2015 [cited 2018 may 25]; 17(3). Available from:

http://dx.doi.org/10.5216/ree.v17i3.31608

23. Souza LD, Lunardi Filho WD. Thofehrn MB. Visibility of nursing work in the context of the clinical model of health care Rev enferm UERJ, Rio de Janeiro, 2015 [cited 2018 may 25]; 23(3):407-12. Available from:

http://www.facenf.uerj.br/v23n3/v23n3al9.pdf DOI: http://dx.doi. org/10.12957/reuerj.2015.6100

24. Miranda NMV, Rewa T, Leonello VM, Oliveira MAC. Advanced practice nursing: a possibility for Primary Health Care? Rev. Bras. Enferm. [Internet]. 2018 [cited 2018 June 01]; 71 (Suppl 1): 716-21. Available from: http://www.scielo.br/scielo.php?script=sci_arttext\&pid=S0034-71672018000700716\&lng=en. http://dx.doi. org/10.1590/0034-7167-2017-0672

25. Rocha EM, Vilela ABA, Oliveira DC, Silva DM, Alves MR, Meira SS. Falily health strategy personnels representational structures regarding domestic violence against older adults. Rev enferm UERJ. [Internet]. 2015 [cited 2018 may 18]; 23(2):178-84. Available from: http://www.facenf.uerj.br/v23n2/ v23n2a06.pdf

26. Souza MFG de, Santos ADB dos, Monteiro A. The nursing process in the opinion of the nursing staff of a teaching hospital. Rev bras enferm. [Internet]. 2013 [cited 2018 may 18]; 66(2): 167-73. Available from: http://www.scielo.br/pdf/reben/ v66n2/03.pdf

27. Marinelli NP, Silva ARA, Silva DNO. Sistematização da assistência de enfermagem -desafios para a implantação. Revista enfermagem contemporânea [Internet]. 2016 [cited 2018 may 23]; 4(2):254-63. Available from: file:///C:/Users/Windows\%207/Downloads/523-3089-1-PB.pdf

28. Soares MI, Resck ZMR, Terra FS, Camelo SHH. Sistematização da assistência de enfermagem: facilidades e desafios do enfermeiro na gerência da assistência. Esc Anna Nery [Internet]. 2015 [cited 2018 may 23]; 19(1):47-53. Available from: http://www.scielo.br/pdf/ean/v19nl/1414-8145ean-19-01-0047.pdf

29. Acioli S, Kebian LVA, Faria MGA, Ferraccioli P. Correa VAF. Práticas do enfermeiro na atenção básica. Rev enferm UERJ [Internet]. 2014 [cited 2018 may 23]: 22(5):637-42. Available from: file:///C:/Users/Windows\%207/Downloads/ 12338-54576-1-PB.pdf

DOI: http://dx.doi.org/10.12957/reuerj.2015.6100 